\title{
Benders' Decomposition Applied to Energy Resource Management in Smart Distribution Networks
}

\author{
J. Soares, Student Member, IEEE, Bruno Canizes, Z. Vale, \\ Senior Member, IEEE \\ GECAD - Research Group on Intelligent Engineering and \\ Computing for Advanced Innovation and Development \\ Polytechnic of Porto, 4200-072, Porto, Portugal \\ \{joaps,brmrc,zav $\} @$ isep.ipp.pt
}

\begin{abstract}
This paper proposes a Benders' decomposition approach for the large-scale Energy Resource Management (ERM). The problem considers large amount of Distributed Energy Resources (DER) including Electric Vehicles (EVs) with gridable capacity. The proposed model is designed for Virtual Power Players/Plants (VPPs) managing a smart distribution network with the aim to maximize profits considering the dayahead time horizon. Previous literature have shown difficulties in solving similar problems using classical techniques in a centralized scheme, namely considering nonlinear network constrains. The proposed approach reduces computational burden of the original Mixed Integer Nonlinear Programming (MINLP) problem. The Benders' cut are introduced for the ERM formulation in order to allow the communication between the slave and the master problem. Analysis of two large-scale instances have been carried out for smart distribution networks, namely a 33-bus and a real Portuguese 180-bus assuming high penetration of DERs and EVs.
\end{abstract}

Index terms: benders' decomposition, energy resource management, smart grid, virtual power player.

\section{INTRODUCTION}

The introduction of Distributed Energy Resources (DER) in distribution networks has motivated the concept of Virtual Power Players (VPP). This entities are designed to raise value for small producers under market-oriented and smart grid environment [1]. One very important tool for VPPs is the Energy Resource Management (ERM) systems to obtain the generation scheduling of DERS at reasonable cost [2], [3].

The multi-period ERM problem is a large-scale Mixed Integer Nonlinear Programming (MINLP) optimization. MINLP techniques require significant computer resources and the computational time is not compatible with the short-term energy scheduling [2].

Decomposition methods can be used when complicating binary variables are identified in optimization problems. An example of decomposition technique is Benders decomposition proposed in 1962 [4]. Benders has been applied successfully to several large-scale nonlinear problems

The present work was done and funded in the scope of the following projects: EUREKA - ITEA2 Project SEAS with project number 12004, UID/EEA/00760/2013 and SFRH/BD/87809/2012 funded by FEDER Funds through COMPETE program and by National Funds through FCT and by NSF (US National Science Foundation) grants: ECCS \#1308192 and IPP $\# 1312260$.

\author{
G. K. Venayagamoorthy, Senior Member, IEEE \\ Real-Time Power and Intelligent Systems Lab. \\ Clemson University, Clemson, SC 29634, USA \\ gkumar@ieee.org
}

[4], [5]. This method is adequate to solve large-scale MINLP problems like the ERM problem. The problem can be divided into a master problem and one or more slave problems. The master problem is usually classified as a linear or mixed integer problem including fewer technical constraints. The slave problems are linear or nonlinear and check if the solution of the master is technically feasible. Benders uses an iterative process which converges when no infeasibilities are found in the slave [4].

Benders' decomposition is proposed to solve the ERM problem for real-size distribution networks with large number of EVs with the aim to reduce computational burden. In [5] authors proposes a multi-objective model for $\mathrm{V} 2 \mathrm{G}$ in smart distribution networks in day-ahead time horizon using Benders to reduce the complexity of the MINLP scheduling problem. However, the sub-problem is formulated as an hourly distribution power flow. Due to temporal dependencies in some variables, we formulate the slave problem as a multiperiod model to obtain better results and we compare with the hourly based approach. Furthermore, the work seems limited in the sense that does not formulate or consider Demand Response (DR) of regular loads, wind, PV generation and Energy Storage Systems (ESS), which are vital features of future smart grids. Also, we improve the model presented in [2] by including ESS, DR, i.e. storage, and Market.

This paper is structured considering the following sections: After introduction, Section II describes the proposed Benders decomposition methodology; Section III illustrates a case study with two scenarios using a 33-bus and a real 180-bus distribution network with the respective results using the proposed approach while some discussion is provided. Finally, the last section presents the main conclusions of the paper.

\section{Methodology}

The multi-period energy resource scheduling formulated in [2] considering network constraints is a MINLP problem. It is our base for the formulation presented here. We improve the model by including Demand Response (DR), Energy Storage Systems (ESS), i.e. storage, and Market. One perspective is considered in this work, i.e. the profit seeking. To calculate 
the profit, $P_{\text {Total }}^{D+1}(1)$, the income, $I_{\text {Total }}^{D+1}(2)$ and costs, $O C_{\text {Total }}^{D+1}$ (3) are calculated for the day-ahead. The profits objective function is achieved by the difference between the income and the total operation costs as can be seen in (1):

$$
\text { Maximize } \quad P_{\text {Total }}^{D+1}=I_{\text {Total }}^{D+1}-O C_{\text {Total }}^{D+1}
$$

To calculate income as expressed in (2): $P_{\text {Load }(L, t)}$ represents the load demand forecast of load $L$ in period $t$ in $\mathrm{kW} ; P_{\operatorname{Sell}(M, t)}$ represents the active power to sell to buyer $M$ in period $t$ in $\mathrm{kW} ; P_{\text {Charge }(E, t)}$ represents the active power charge of ESS $E$ in period $t$ in $\mathrm{kW} ; P_{\text {Charge }(V, t)}$ represents the active power charge of EV $V$ in period $t$ in $\mathrm{kW} ; M P_{\text {Load }(L, t)}$ represents the price that load $L$ pays in period $t$ in m.u.; $M P_{S e l l(M, t)}$ represents the price that buyer $M$ pays in period $t$ in m.u.; $M P_{\text {Charge }(E, t)}$ represents the price that the ESS $E$ pays to charge in period $t$ in m.u.; represents the price that $\mathrm{EV} V$ pays to charge in period $t$ in m.u.

$$
\begin{aligned}
& \text { Maximize } \quad I_{\text {Total }}^{D+1}=
\end{aligned}
$$

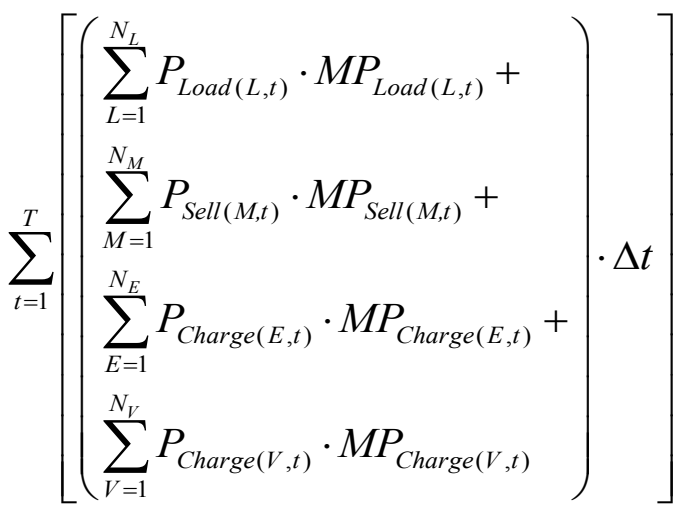

The operation costs of the VPP can be expressed as in (3):

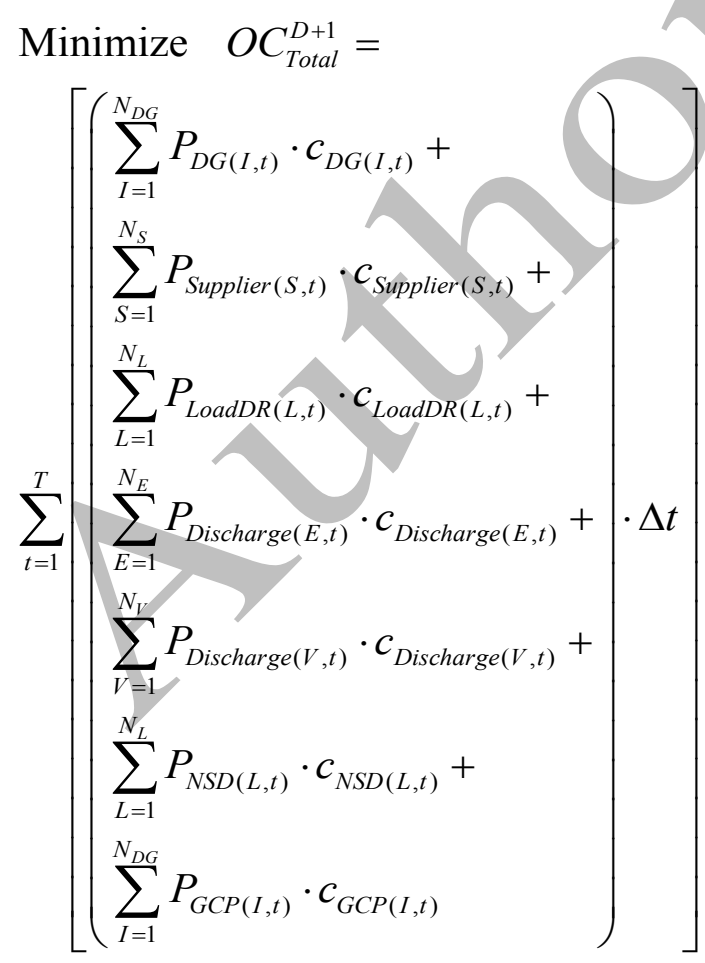

$P_{D G(I, t)}$ represents the active power generation of DG unit $I$ in period $t$ in $\mathrm{kW} ; P_{\text {Supplier }(S, t)}$ represents the active power bought to external supplier $S$ in period $t$ in $\mathrm{kW} ; P_{\operatorname{LoadDR}(L, t)}$ represents the active power reduction of load $L$ in period $t$ in $\mathrm{kW} ; P_{\text {Discharge }(E, t)}$ represents the active power discharge of ESS $E$ in period $t$ in $\mathrm{kW} ; P_{\text {Discharge }(V, t)}$ represents the active power discharge of $\mathrm{EV} V$ in period $t$ in $\mathrm{kW} ; P_{N S D(L, t)}$ represents the active power of non-supplied demand of load $L$ in period $t$ in $\mathrm{kW} ; P_{C G P(I, t)}$ represents the generation curtailment power of DG unit $I$ in period $t$ in $\mathrm{kW} ; C_{D G(I, t)}$ represents the generation cost of DG unit $I$ in period $t$ in m.u.; $C_{\text {Supplier }(S, t)}$ represents the electricity cost of external supplier $S$ in period $t$ in m.u.; $C_{\text {LoadDR(L,t) }}$ represents the load reduction (DR) cost of load $L$ in period $t$ in m.u.; $C_{\text {Discharge(E,t) }}$ represents the discharging cost of ESS $E$ in period $t$ in m.u.; $C_{\text {Discharge }(V, t)}$ represents the discharging cost of EV $V$ in period $t$ in m.u.; $C_{N S D(L, t)}$ represents the non-supplied demand cost of load $L$ in period $t$ in m.u.; $C_{C G P(I, t)}$ represents the curtailment cost of DG unit $I$ in period $t$ in m.u.

The mathematical model is also subjected to several constraints, namely the network constraints, the distributed generation maximum and minimum powers, the ESS technical constraints, EVs and DR. More details can be found in [2], [6].

\section{A. Benders approach}

The MINLP optimization techniques require high execution time to deal with the ERM for real size distribution networks with large number of EVs. Benders decomposition is implemented in this work to overcome the difficulties to solve nonlinear optimization with discrete variables [4]. In fact, two approaches are implemented in this paper to solve the ERM problem, namely a multi-period approach and an hourly power flow approach, named here as Benders-hourly. We propose the multi-period approach because the hourly power flow approach cannot deal with the temporal dependencies arise with ESS and EVs, e.g. not be able to see minimum energy requirements ahead, thus resulting in sub-optimal solutions. Nevertheless, we use Benders-hourly as an effective and lighter approach, but we demonstrate that Benders multiperiod finds better solutions. Figure 1 presents the flowchart of the proposed multi-period approach.

In (4) the linear Benders cuts are formulated for the problem under study where $Z_{u p}$ represents the sum of infeasibilities of the slave problem; the index $m$ represents the current iteration and the $m-1$ the results of previous iteration; $\lambda_{\text {Supplier(S,t) }}^{m-1}$ represents the Lagrange of the associated constraint of supplier $S$ in period $t ; \lambda_{D G(I, t)}^{m-1}$ represents the Lagrange of the associated constraint of DG unit $I$ in period $t$; $\lambda_{E S S_{-} d s c(E, t)}^{m-1}$ and $\lambda_{E S S_{-} \operatorname{chg}(E, t)}^{m-1}$ represents the Lagrange of the associated constraint of ESS $E$ in period $t ; \lambda_{E V_{-} d s c(V, t)}^{m-1}$ and $\lambda_{E V \_}^{m-1} \operatorname{chg}(V, t)$ represents the Lagrange of the associated constraint of EV $V$ in period $t ; X_{S u p p l i e r(S, t)}^{m}$ represents the binary variable of Supplier $S$ in period $t$ in iteration $m$; 
$X_{\text {Supplier }(S, t)}^{m-1}$ represents the previous results of binary variable of Supplier $S$ in period $t$ in iteration $m-1 ; X_{D G(I, t)}^{m}$ represents the binary variable of DG unit $I$ in period $t$ in iteration $m$; $X_{D G(I, t)}^{m-1}$ represents the results of the binary variable of DG unit $I$ in period $t$ in iteration $m-1 ; X_{E S S(E, t)}^{m}$ represents the discharging binary variable of ESS $E$ in period $t$ in iteration $m ; X_{E S S(E, t)}^{m-1}$ represents the results of the discharging binary variable of ESS $E$ in period $t$ in iteration $m-1 ; Y_{E S S(E, t)}^{m}$ represents the charging binary variable of ESS $E$ in period $t$ in iteration $m ; Y_{E S S(E, t)}^{m-1}$ represents the results of the charging binary variable of ESS $E$ in period $t$ in iteration $m-1 ; X_{E V(V, t)}^{m}$ represents the discharging binary variable of $\mathrm{EV} V$ in period $t$ in iteration $m ; X_{E V(V, t)}^{m-1}$ represents the results of the discharging binary variable of EV $V$ in period $t$ in iteration $m$ $1 ; Y_{E V(V, t)}^{m}$ represents the charging binary variable of $\mathrm{EV} V$ in period $t$ in iteration $m ; Y_{E V(V, t)}^{m-1}$ represents the results of the charging binary variable of EV $V$ in period $t$ in iteration $m-1$. The Lagrange values are obtained in the slave problem of the previous iteration.

$$
\begin{aligned}
& \alpha^{*} \geq Z_{u p}^{m-1}+ \\
& \sum_{t=1}^{T} \sum_{S}^{N_{S}} \lambda_{\text {Supplier }(S, t)}^{m-1} \cdot\left(X_{\text {Supplier }(S, t)}^{m}-X_{S u p p l i e r(S, t)}^{m-1}\right)+ \\
& \sum_{t=1}^{T} \sum_{I \in \Omega_{D G}^{d}} \lambda_{D G(I, t)}^{m-1} \cdot\left(X_{D G(I, t)}^{m}-X_{D G(I, t)}^{m-1}\right)+ \\
& \sum_{t=1}^{T} \sum_{E=1}^{N_{E}} \lambda_{E S S_{-} d s c(E, t)}^{m-1} \cdot\left(X_{E S S(E, t)}^{m}-X_{E S S(E, t)}^{m-1}\right)+ \\
& \sum_{t=1}^{T} \sum_{E=1}^{N_{E}} \lambda_{E S S_{-} \operatorname{chg}(E, t)}^{m-1} \cdot\left(Y_{E S S(E, t)}^{m}-Y_{E S S(E, t)}^{m-1}\right)+ \\
& \sum_{t=1}^{T} \sum_{V=1}^{N_{V}} \lambda_{E V_{-} d s c(V, t)}^{m-1} \cdot\left(X_{E V(V, t)}^{m}-X_{E V(V, t)}^{m-1}\right)+ \\
& \sum_{t=1}^{T} \sum_{V=1}^{N_{V}} \lambda_{E V_{-} \operatorname{chg}(V, t)}^{m-1} \cdot\left(Y_{E V(V, t)}^{m}-Y_{E V(V, t)}^{m-1}\right)
\end{aligned}
$$

The benders cut are generated in each iteration and added to the master problem. The original objective function (3) is changed to include the alpha $(\alpha)$ term, i.e. the master tries to minimize the value of alpha but satisfying the added constraints (4). In the slave problem the original objective function (3) is changed to (5) where $Z A_{b, t,,} Z Q_{b, t,} Z F_{b k, t}$, represents the relaxation variables (also called slack variables), namely the relaxation for nonlinear power flow of active and reactive powers in each bus $b$, and the capacity of each line $b k$. The value of these variables represents how much some constraint is being violated, e.g. exceeded capacity in the line. In fact, the slave problem cannot change the binary variables but is free to explore the continuous variables in order to satisfy the several constraints while minimizing the objective and the value of the slack variables.

$$
\begin{array}{ll}
\text { Minimize } & P_{\text {Total }}^{D+1}+\sum_{t=1}^{T} \sum_{b=1}^{N_{B}}\left(Z A_{b, t}+Z Q_{b, t}\right)+ \\
\sum_{t=1}^{T} \sum Z F_{b k, t} & k \neq b
\end{array}
$$

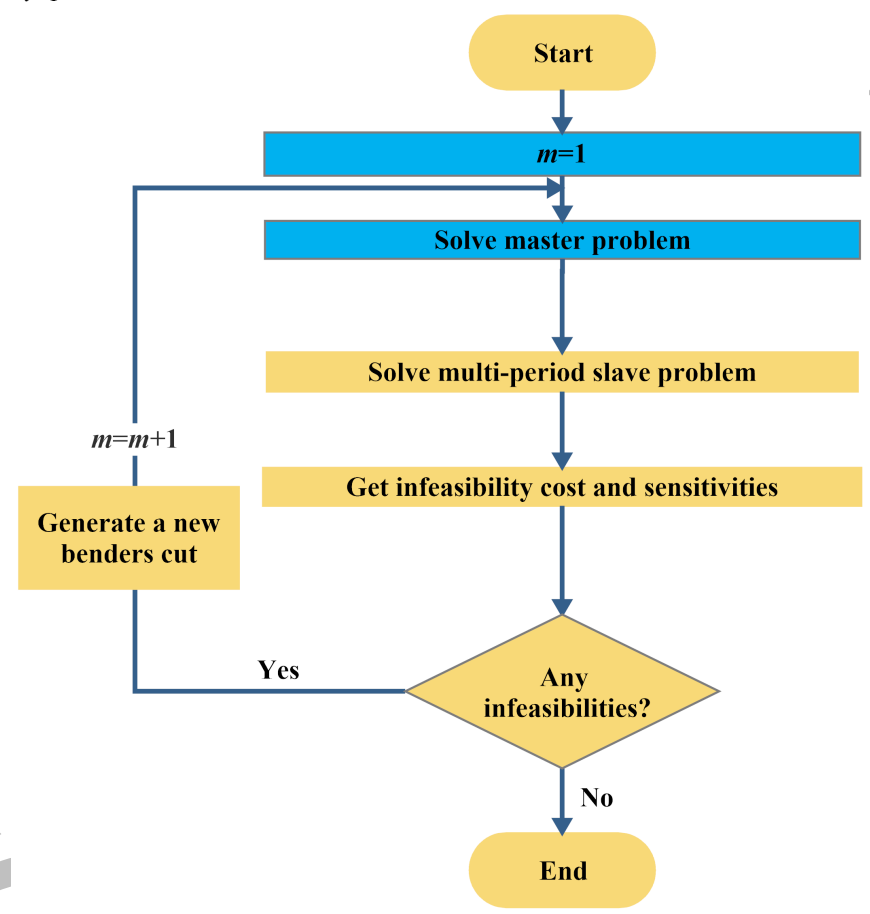

Figure 1. Proposed Benders approach flowchart

The iterative methods like the proposed approach requires a stopping criteria in order to come to a stop. The algorithm ends when the difference between $Z_{u p}$ and $Z_{\text {down }}$ is equal to zero or lower than a very small value. The $Z_{\text {down }}$ represents the value of the obtained cuts in the master problem, i.e. the value of alpha. If $Z_{\text {down }}$ jumps to a higher value than $Z_{u p}$ it might indicate bad performance of the optimization. The convergence often occurs in the first iteration when the solution of the master problem is feasible.

\section{CASE STUDIES}

Two case studies are developed to demonstrate how the proposed approach performs. The tests are performed in two different network systems, namely a 33-bus $12.66 \mathrm{kV}$ distribution test network adapted from [7] and a 180-bus 30 $\mathrm{kV}$ real distribution network from Portugal [8]. The 33-bus test system is a $12.66 \mathrm{kV}$ network with one substation, 32 MV load points, $1800 \mathrm{EVs}, 67$ DGs including a large wind unit, 10 external suppliers and 15 ESSs. A variant of this case with 180-bus is also discussed. The 180-bus test system is a real Portuguese distribution $30 \mathrm{kV}$ network with one substation adapted to a future scenario, $90 \mathrm{MV}$ load points, 6000 EVs, 116 DG units, 1 external supplier, and 7 ESSs. The external suppliers are modeled in bus 1 corresponding to the substation location and connection with the main grid. In 
both test cases Demand Response (DR) with Direct Load Control (DLC) is considered. The benefit for DLC contracts is set to $0.02 \mathrm{~m} . \mathrm{u} . / \mathrm{kWh}$ in both cases. The consumers receive this amount per each reduced $\mathrm{kWh}$ energy. The charging price of EVs and ESS is 0.15 m.u. $/ \mathrm{kWh}$ in both cases. The ESS initial state of charge are set to empty in the simulations. The selling energy price, i.e. energy sold to external entities, is considered 0.14 m.u. $/ \mathrm{kWh}$ in both cases. The considered prices and the DG capacity of the test systems have taken into account the observations made in the projections of [9]. The scenarios of EVs for both systems have been developed using the tool presented in [8]. The details of each test system are described in the following subsections. In both cases, the goal of the VPP is to maximize the profits.

The work was developed in MATLAB R2014a 64 bits, GAMS, and TOMLAB 64 bits software [10] using a computer with one Intel Xeon E5-1650 processor and 6 GB of RAM running Windows 8.1. The solvers used in TOMLAB were SNOPT and CPLEX and in GAMS, the DICOPT which is based on CONOPT and CPLEX solvers.

\section{A. 33-bus scenario}

For the 33-bus test system a fleet of 1800 EVs with V2G capabilities is considered. The energy demand predicted for the trips is equal to $13.77 \mathrm{MWh}$ corresponding to an average of $7.65 \mathrm{kWh}$ per vehicle and a total of 2553 trips (average of 1.4 trips per vehicle) over the entire day. It is assumed that the EVs require at least $30 \%$ of the battery capacity in the end of the day. The consumers have to pay the VPP the contracted supply price, which is $0.14 \mathrm{~m} . \mathrm{u} . / \mathrm{kWh}$. The charging price for EVs and ESS is 0.14 m.u. $/ \mathrm{kWh}$. The discharging cost considered for the EVs and ESS is set to $0.19 \mathrm{~m} . \mathrm{u} . / \mathrm{kWh}$ in this scenario. A charging/discharging efficiency of $77 \%$ is considered for EVs and $90 \%$ for ESS. The initial state of charge of EVs battery are randomly initialized in this scenario. The total capacity from the available external suppliers is $6.20 \mathrm{MW}$ in the considered time horizon. The single-line diagram of the 33-bus distribution network can be seen in Figure 2. Table I presents the considered costs and the capacity of the 33-bus system. The total forecasted regular load without EVs, ESS and DR is 102.97 MWh.

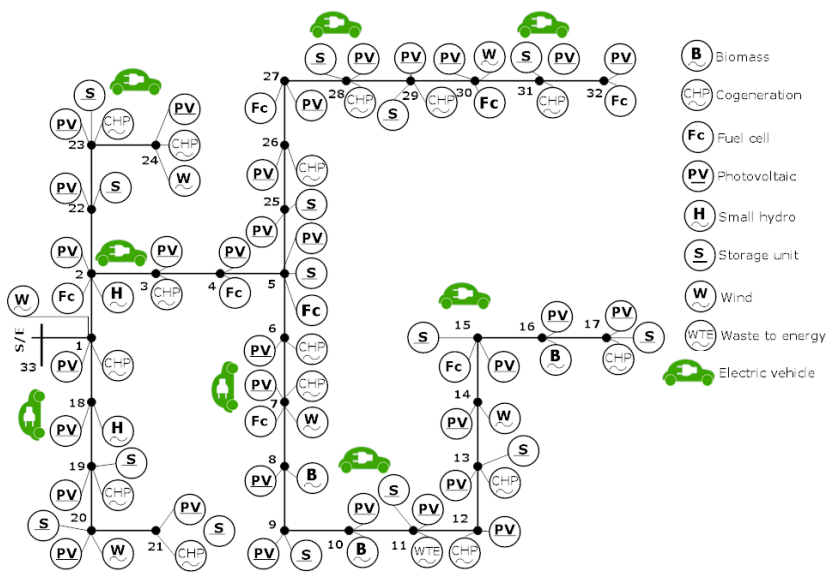

TABLE I. 33-BUS GRID SCENARIO CHARACTERIZATION

\begin{tabular}{c|c|c|c|c|c}
\hline \hline $\begin{array}{c}\text { Energy } \\
\text { resources }\end{array}$ & $\begin{array}{c}\text { Min. price } \\
(\boldsymbol{m} . \boldsymbol{u} \cdot / \boldsymbol{k} \boldsymbol{W h})\end{array}$ & $\begin{array}{c}\text { Max. price } \\
(\boldsymbol{m} . \boldsymbol{u} . / \boldsymbol{k} \boldsymbol{W h})\end{array}$ & $\begin{array}{c}\text { Min. } \\
\text { capacity } \\
(\boldsymbol{M} \boldsymbol{W})\end{array}$ & $\begin{array}{c}\text { Max. } \\
\mathbf{c a p a c i t y} \\
(\boldsymbol{M} \boldsymbol{W})\end{array}$ & $\begin{array}{c}\text { Units } \\
\#\end{array}$ \\
\hline \hline Biomass & 0.09 & 0.09 & 0.38 & 0.38 & 4 \\
\hline CHP & 0.06 & 0.06 & 1.15 & 1.15 & 15 \\
\hline Fuel Cell & 0.15 & 0.15 & 0.11 & 0.11 & 7 \\
\hline $\begin{array}{c}\text { Small } \\
\text { Hydro }\end{array}$ & 0.07 & 0.07 & 0.07 & 0.07 & 2 \\
\hline PV & 0.20 & 0.20 & 0.00 & 0.84 & 31 \\
\hline $\begin{array}{c}\text { Waste-to- } \\
\text { energy }\end{array}$ & 0.10 & 0.10 & 0.01 & 0.01 & 1 \\
\hline Wind & 0.15 & 0.15 & 0.18 & 0.89 & 6 \\
\hline $\begin{array}{c}\text { Large } \\
\text { Wind }\end{array}$ & 0.07 & 0.07 & 1.58 & 1.80 & 1 \\
\hline $\begin{array}{c}\text { External } \\
\text { Suppliers }\end{array}$ & 0.09 & 0.30 & 6.20 & 6.20 & 10 \\
\hline V2G & 0.19 & 0.19 & 0.00 & 5.72 & 1800 \\
\hline ESS & 0.19 & 0.19 & 0.90 & 0.90 & 15 \\
\hline DR & 0.16 & 0.16 & 0.60 & 1.17 & 32 \\
\hline \hline
\end{tabular}

B. 180-bus scenario

In the 180-bus scenario the fleet of EVs considered is 6000 EVs. The total energy demand predicted for the trips is 34.26 MWh corresponding to an average of $5.7 \mathrm{kWh}$ per vehicle and a total of 10137 trips (average of 1.7 trips per vehicle). The charging cost considered for EVs and ESS is 0.15 m.u. $/ \mathrm{kWh}$ and $0.13 \mathrm{~m}, \mathrm{u} . / \mathrm{kWh}$, respectively. The discharging cost considered for the EVs and ESS is set to 0.19 m.u./kWh and $0.18 \mathrm{~m} . \mathrm{u} . / \mathrm{kWh}$, respectively. It is assumed that the discharging price covered the battery degradation costs. A charging/discharging efficiency of $90 \%$ is considered for EVs and ESS. The initial state of charge of EVs battery are initialized to $30 \%$. Only one external supplier is available and the contract is $10 \mathrm{MW}$, with a minimum of $2 \mathrm{MW}$ purchase in the considered time horizon. The single-line diagram of the 180-bus distribution network can be seen in Figure 3. Table II presents the considered costs and the capacity of the system. The total forecasted regular load without EVs, ESS and DR is 243.36 MWh. The minimum forecasted wind power is 1.07 MW and the maximum is $1.76 \mathrm{MW} .17$ units of biomass are dispatchable assuming that there is plenty of burning resources available, thus proving a constant capacity of 1.98 MW. The ESS are composed of 7 batteries with an energy storage capacity ranging from $500-1000 \mathrm{kWh}$ and a maximum charging/discharging rate between $100-200 \mathrm{~kW}$. The contracted DR capacity ranges from 2.47- 4.26 MW.

TABLE II. 180-BUS GRID SCENARIO CHARACTERIZATION

\begin{tabular}{c|c|c|c|c|c}
\hline \hline $\begin{array}{c}\text { Energy } \\
\text { resources }\end{array}$ & $\begin{array}{c}\text { Min. price } \\
(\boldsymbol{m} . \boldsymbol{u} \cdot \boldsymbol{k} \boldsymbol{k} \boldsymbol{W h})\end{array}$ & $\begin{array}{c}\text { Max. price } \\
(\boldsymbol{m} . \boldsymbol{u} \cdot \boldsymbol{k} \boldsymbol{k} \boldsymbol{W h})\end{array}$ & $\begin{array}{c}\text { Min. } \\
\text { capacity } \\
(\boldsymbol{M W})\end{array}$ & $\begin{array}{c}\text { Max. } \\
\text { capacity } \\
(\boldsymbol{M} \boldsymbol{W})\end{array}$ & $\begin{array}{c}\text { Units } \\
\#\end{array}$ \\
\hline \hline PV & 0.15 & 0.15 & 0.00 & 1.49 & 44 \\
\hline Wind & 0.09 & 0.09 & 1.07 & 1.76 & 55 \\
\hline Biomass & 0.13 & 0.13 & 1.98 & 1.98 & 17 \\
\hline $\begin{array}{c}\text { External } \\
\text { supplier }\end{array}$ & 0.10 & 0.16 & 10.00 & 10.00 & 1 \\
\hline V2G & 0.19 & 0.19 & 19.05 & 19.05 & 6000 \\
\hline ESS & 0.18 & 0.18 & 1.20 & 1.20 & 7 \\
\hline DR & 0.18 & 0.18 & 2.47 & 4.26 & 90 \\
\hline \hline
\end{tabular}

Figure 2. Single-line diagram of 33-bus network 


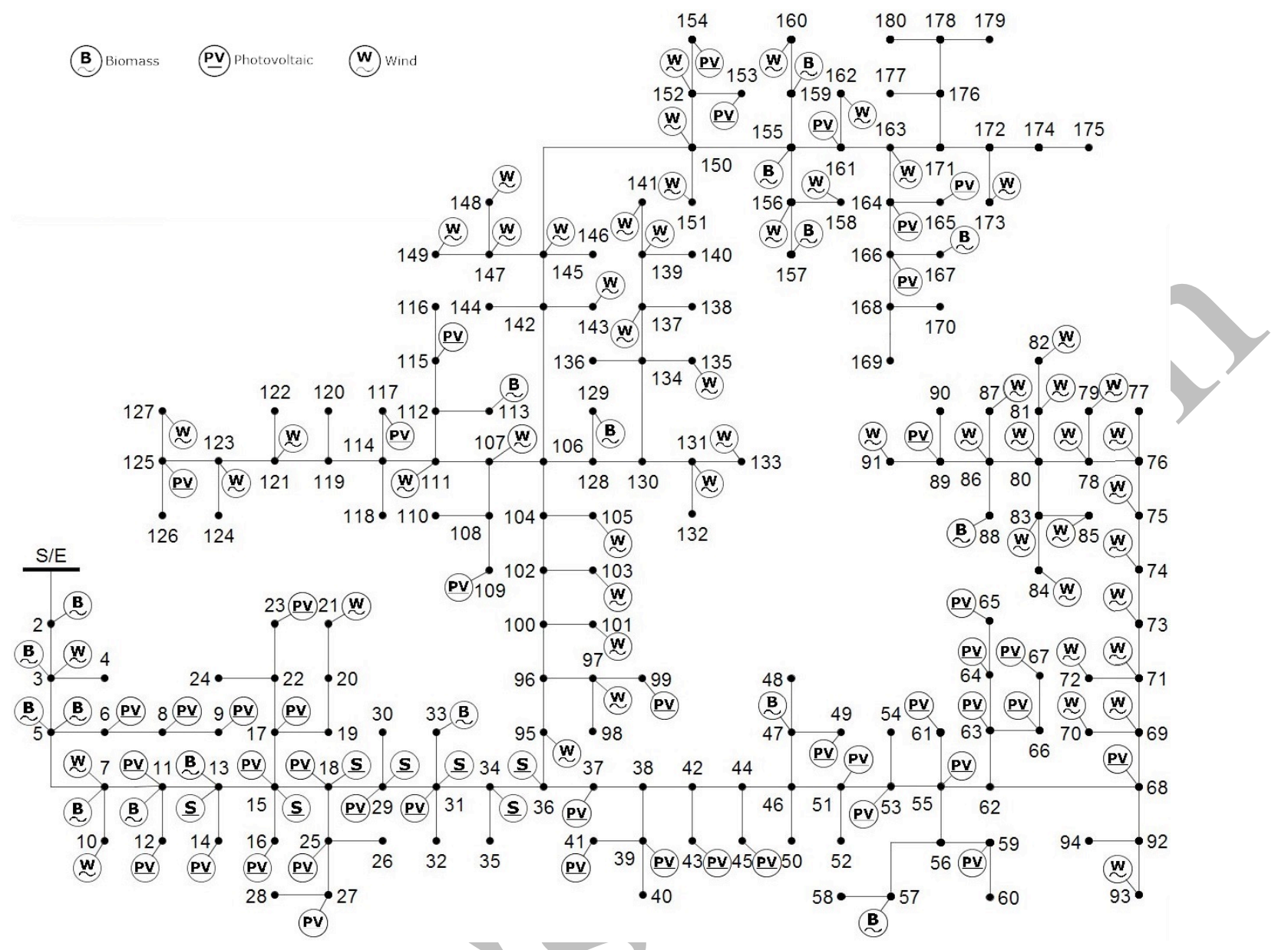

Figure 3. Single-line diagram of 180 -bus network

\section{RESULTS AND DISCUSSION}

The Benders' method is applied to two large-scale cases: a 33-bus network with 32 aggregated loads, 66 DGs, 15 storage units and 1800 individual EVs; and a 180-bus real Portuguese network with 90 aggregated loads, 116 DGs, 7 storage units and 6000 individual EVs.

To compare the performance of the implemented approach, we also included the hourly Benders approach, MILP and MINLP. The MILP approach uses a relaxation of the original formulation without including the optimal distribution power flow.

Table III and IV summarizes the obtained results regarding the value of the objective function (profits in m.u.), the computational peak memory and the execution time for Benders, MILP and MINLP techniques. The obtained profits are higher in MILP because the power losses are not considered. It is possible to see that similar results are obtained with the proposed Benders when compared with MINLP but at reduced computational effort. In fact, the proposed approach is better when compared with Bendershourly approach in both cases. Tough, execution time is worse for the proposed approach when compared with Benders-hourly. However, the amount of execution time taken by the full mathematical model is not acceptable for the available timeframe in the decision making process.
Nevertheless, the proposed approach obtains a very close solution in much less time when compared with MINLP, from 323 hours to just 1.5 hours. To evaluate the impact on computer system resources a memory test was made using MATLAB memory profiler tool for the 33-bus and 180-bus scenarios. This method reported the peak memory for each function. In the 33-bus scenario, the peak memory use reported for MILP approach using TOMLAB and Benderhourly was $52 M B$ while Benders multi-period approach was $190 M B$. GAMS memory used was measured by analyzing Windows memory information and was around $270 \mathrm{MB}$ using MINLP. Regarding the 180-bus scenario, the peak memory use was considerable higher, namely $164 M B$ in MILP and Benders-hourly, $629 \mathrm{MB}$ in Benders multi-period approach and $1080 \mathrm{MB}$ in GAMS for MINLP.

Regarding problem dimension in the 180-bus scenario, the MILP relaxed formulation takes 745,033 variables by 870,754 linear constraints. Of these variables, 291,114 are integer variables. In the slave problem the number of variables is reduced to 476,928 variables by 427,522 constraints (21,528 nonlinear constraints). In the hourly approach each sub-problem is much smaller, namely with 14,342 variables, 1428 constraints of which 897 are nonlinear constraints. 
TABLE III. COMPARISON IN 33-BUS SCENARIO - 1800 EVS

\begin{tabular}{c|c|c|c}
\hline \hline Method & Profits (m.u.) & $\begin{array}{c}\text { Peak memory } \\
(\boldsymbol{M B})\end{array}$ & $\begin{array}{c}\text { Execution } \\
\text { time }\end{array}$ \\
\hline \hline Benders & 5653 & 190 & 15 minutes \\
\hline Benders-hourly & 5625 & 52 & 50 seconds \\
\hline MILP & 5854 & 52 & 8 seconds \\
\hline MINLP & 5666 & 270 & 14 hours \\
\hline \hline
\end{tabular}

TABLE IV. COMPARISON IN 180-BUS SCENARIO - 6000 EVS

\begin{tabular}{c|c|c|c}
\hline \hline Method & Profits (m.u.) & $\begin{array}{c}\text { Peak memory } \\
(\boldsymbol{M B})\end{array}$ & $\begin{array}{c}\text { Execution } \\
\text { time }\end{array}$ \\
\hline \hline Benders & 3348 & 629 & 100 minutes \\
\hline Benders-hourly & 3237 & 164 & 160 seconds \\
\hline MILP & 3773 & 164 & 35 seconds \\
\hline MINLP & 3365 & 1080 & 323 hours \\
\hline \hline
\end{tabular}

Figure 4 presents the obtained energy resources scheduling using Benders decomposition approach as proposed by this paper. It is possible to see that during night periods a high energy consumption is expected due to EVs charging. On another hand, during periods where external suppliers are costlier ("used to be" peak periods), DR and EVs discharging are activated in an intelligent manner to sustain the demand and achieve a higher profit for the aggregator, namely in periods 12 to 14 and 17 to 21 . The external suppliers deliver $55 \%$ of the energy requested during the day, while DG, DR and EVs, $31 \%, 9 \%$ and $5 \%$ respectively. The EVs account for $18 \%$ of the energy consumed in this situation. The ESS are not used according to the results of the scheduling algorithm.

In a second experiment, ESS charging price was changed to $0.15 \mathrm{~m} . u . / \mathrm{kWh}$. Figure 5 and Figure 6 reveals the impact of this change in the energy resources scheduling. It can be seen thatthe storage charges in night hours while energy from external supplier is cheaper and then discharges when energy is more expensive for the aggregator. In this case, the external supplier delivers $54 \%$, while DG, DR, EVs and ESS, 31\%, $10 \%, 3 \%$ and $2 \%$ of the energy production needs, respectively. The EVs charging account for $15 \%$ of the total energy demand while ESS charging for 3\%. In this case, the profit increased 51 m.u. from 3237 m.u. to 3288 m.u. in Benders-hourly approach while in Benders proposed approach increased $78 m$.u. from $3348 m . u$. to $3426 m . u$. The power losses achieved in the proposed Benders approach reduced to $2.51 \mathrm{MWh}$ from $2.65 \mathrm{MWh}$ in the previous experiment with different charging price. The value of power loses was higher in other methods except from MINLP.

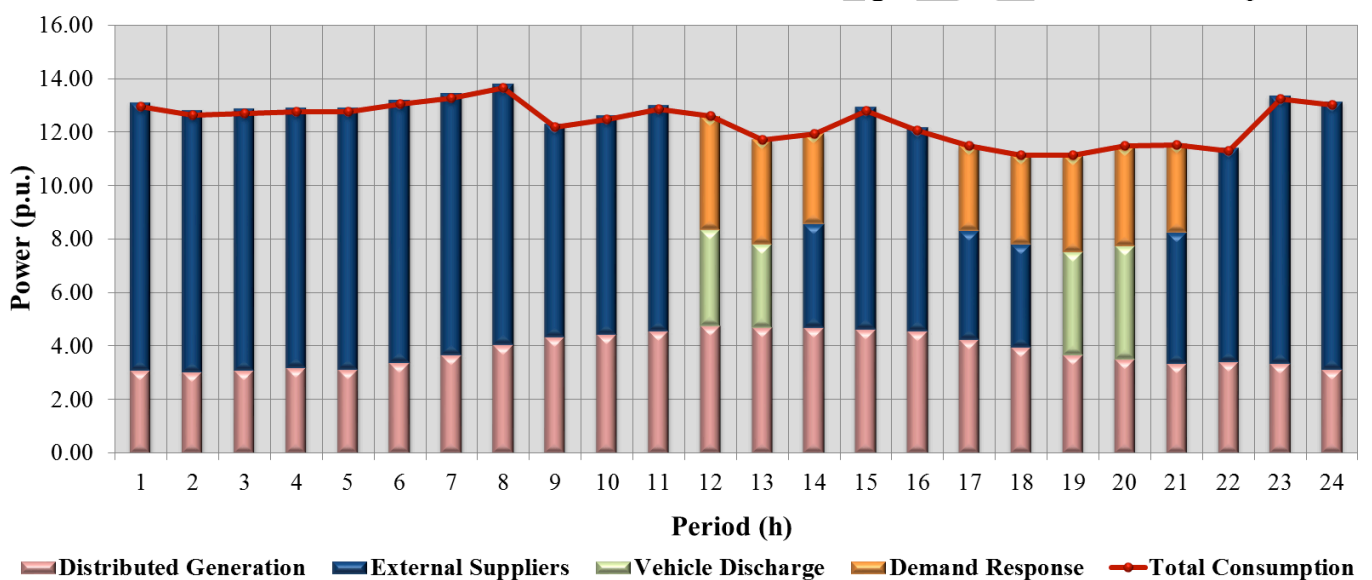

$\square$ Distributed Generation $\square$ External Suppliers $\square$ Vehicle Discharge $\square$ Demand Response $\sim$ Total Consumption

Figure 4. Energy resources scheduling of 180-bus scenario

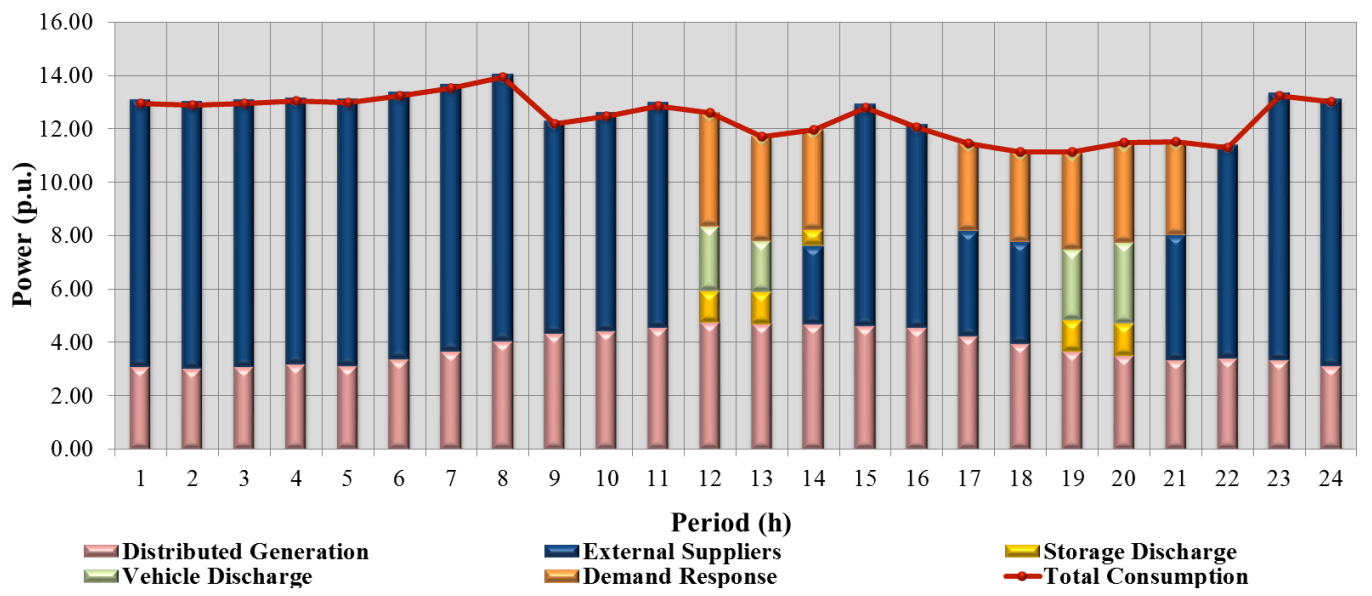

Figure 5. Energy resources scheduling of 180-bus scenario: ESS charging price of 0.15 m.u. $/ \mathrm{kWh}$ 


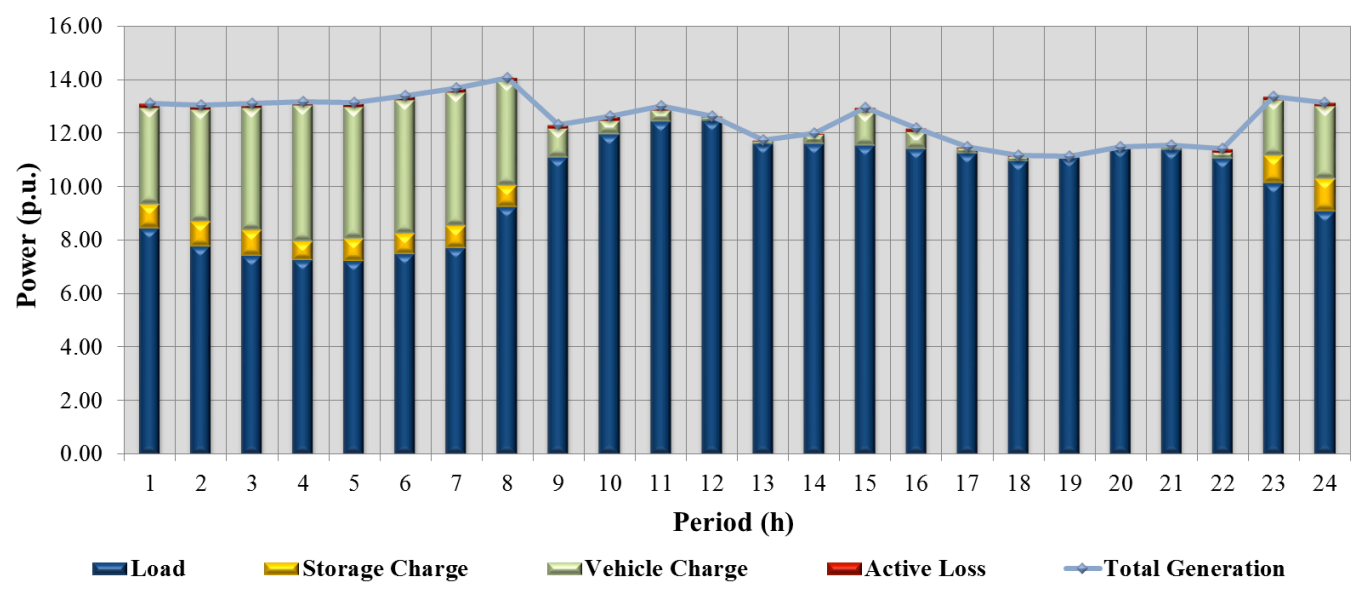

Figure 6. Consumption scheduling of 180-bus scenario: ESS charging price of 0.15 m.u. $/ \mathrm{kWh}$

\section{A. Discussion}

The obtained results indicate that the proposed approach is suitable for ERM problems. In fact, the tests realized in the case study have real-world problem dimension. When compared with the hourly approach, the multi-period approach is somewhat heavier and less scalable. However, the execution time is still very reasonable for the decision making process. It should be take into account that tests were executed in a standard workstation and not on a faster dedicated server. More importantly, the objective function achieved is better in both cases. In 180-bus scenario this value is higher than $100 \mathrm{~m} . \mathrm{u}$. in both experiments. Thus, assuming similar performance and same situation over an entire year, the profits could increase by around 40,000 m.u., in other words corresponding to a total profit of 10 days. Compared with the MINLP approach, the results indicate the proposed Benders is only worse by $0.2-0.5 \%$. The MILP approach is the fastest approach in the test but not includes power losses in the formulation. However, it can be used as initial solution to other methods, such as metaheuristics, MINLP or Benders. MILP could be adequate when the network constraints are not important or when an approximate solution is needed.

An important remark is that an increased level of flexibility, i.e. high number of energy resources, can improve the convergence of the Benders approaches. These resources can contribute because in the slave problem (NLP) a feasible solution can be found by adjusting some continuous variables (binaries are not changed) in order to satisfy the nonlinear constraints. With less flexibility it becomes more difficult to attain it.

In the 180-bus scenario we increased the charging price of ESS showing that the algorithm effectively chooses the adequate resources according to the objective. With a higher price the VPP charged the ESS during night (more income) and used them to provide energy (at less costs) later. With lower price the ESS were not used because did not provide added profit by using them. This was possible to conclude because the state of charge of the ESS was initially set to empty. This demonstrates that the business model for ESS in microgrids and smart distribution networks should be carefully investigated. Alternatively, the ESS can belong to the DSO or be part of the assets of the VPP/aggregator which we do not address here (out of the scope of the research) but could be easily done with our model by changing the price schemes or removing the ESS terms from the objective function.

As with every other model, the proposed approach as some limitations. The most important is that it relies on forecast inputs that should be as accurate as possible. Considering several scenarios with an associated probability could be a future path.

\section{CONCLUSIONS}

Benders' approach appears to be a competitive method for large-scale ERM problems. The proposed approach could achieve almost the same result in one hour and half than a classical approach do in 13 days. Future work will address ways to consider uncertainty of wind and PV power in the ERM incorporating optimal distribution power flow for realworld applications by using strategies like the one presented here. Currently, MINLP classic approaches are only practicable in small instances.

\section{REFERENCES}

[1] S. Y. S. You, C. Traeholt, and B. Poulsen, "A market-based Virtual Power Plant," in 2009 International Conference on Clean Electrical Power, 2009, pp. 460-465.

[2] J. Soares, T. Sousa, H. Morais, Z. Vale, B. Canizes, and A. Silva, "Application-Specific Modified Particle Swarm Optimization for energy resource scheduling considering vehicle-to-grid," Appl. Soft Comput., vol. 13, no. 11, pp. 4264-4280, 2013.

[3] A. Y. Saber and G. K. Venayagamoorthy, "Resource Scheduling Under Uncertainty in a Smart Grid with Renewables and Plug-in Vehicles," IEEE Syst. J., vol. 6, no. 1, pp. 103-109, 2012.

[4] A. J. Conejo, E. Castillo, R. Minguez, and R. Garcia-Bertrand, Decomposition Techniques in Mathematical Programming: Engineering and Science Applications. Springer, 2006.

[5] A. Zakariazadeh, S. Jadid, and P. Siano, "Multi-objective scheduling of electric vehicles in smart distribution system," Energy Convers. Manag., vol. 79, pp. 43-53, Mar. 2014. 
[6] H. Morais, T. Sousa, J. Soares, P. Faria, and Z. Vale, "Distributed energy resources management using plug-in hybrid electric vehicles as a fuel-shifting demand response resource," Energy Convers. Manag., vol. 97, no. June 2015, pp. 79-93, 2015.

[7] M. E. Baran and F. F. Wu, "Network Reconfiguration in DistributionSystems for Loss Reduction and Load Balancing," IEEE Trans. Power Deliv., vol. 4, no. 2, pp. 1401-1407, 1989.

[8] J. Soares, B. Canizes, C. Lobo, Z. Vale, and H. Morais, "Electric Vehicle Scenario Simulator Tool for Smart Grid Operators," Energies, vol. 5, no. 6, pp. 1881-1899, 2012.
[9] A. Zervos, C. Lins, and J. Muth, RE-thinking 2050: a 100\% renewable energy vision for the European Union. EREC, 2010.

[10] TOMLAB, “TOMLAB optimization," 2015. [Online]. Available: http://tomopt.com/tomlab/about/.

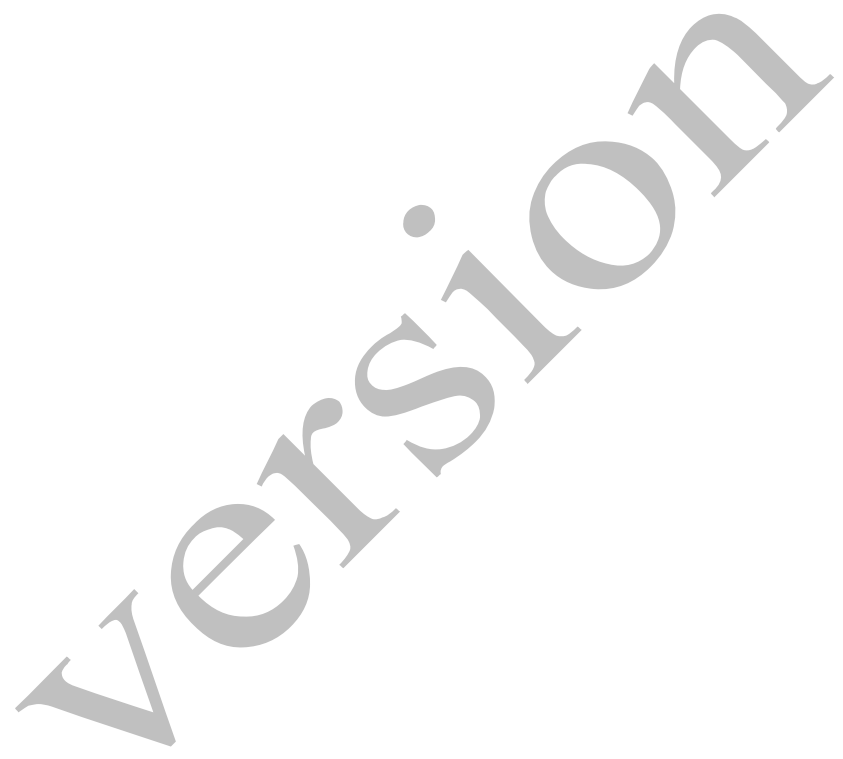

